\title{
lieuxdits \#17
}

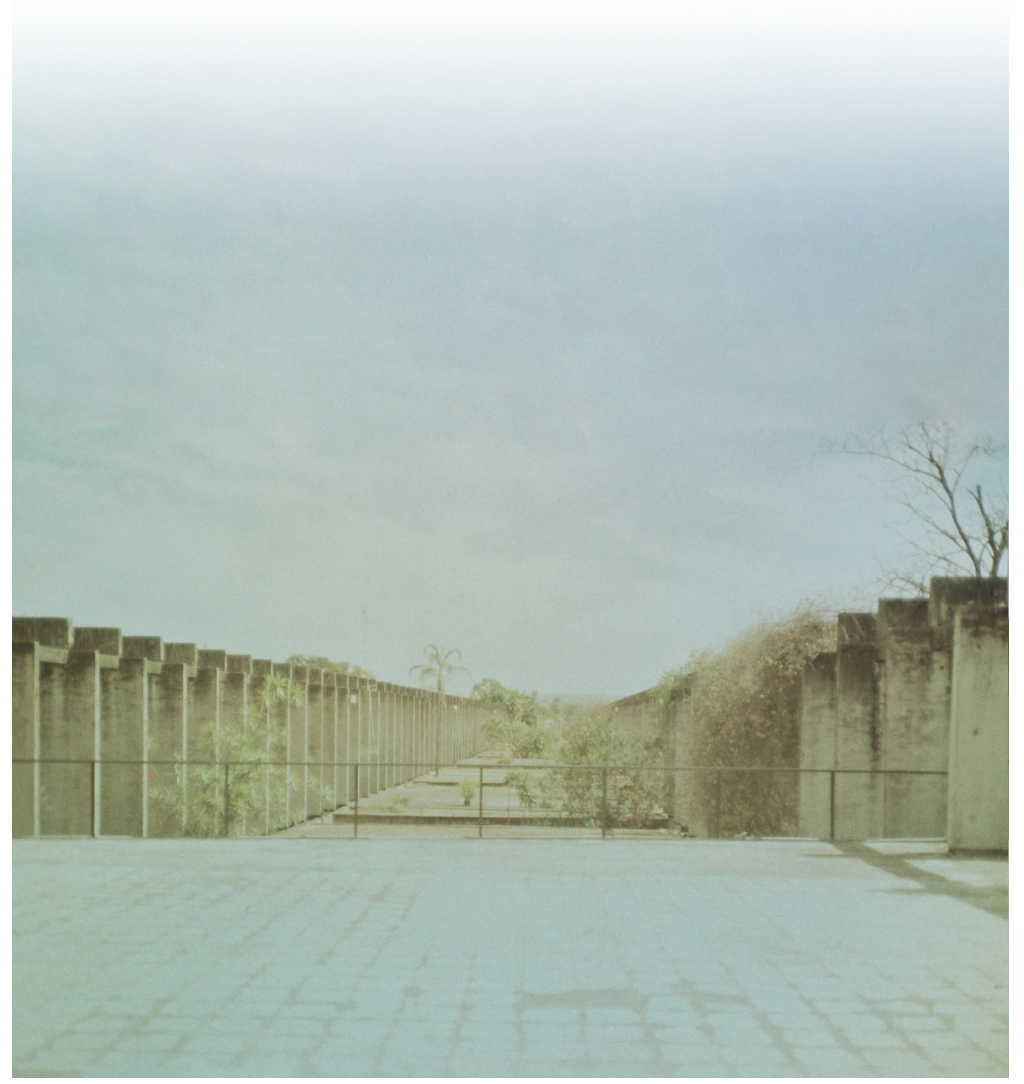

Référence bibliographique :

Catherine Lembrée, Jonas Hanssens,

Aurélien Hucq, Fiorella Quadu - co-auteurs ; Yves Hanin et Charles-Hubert Born - promoteurs de la recherche, "Gestion dynamique de la nature temporaire en Région bruxelloise", lieuxdits\#17, décembre 2019, pp. 12-15.

\section{La revue lieuxdits}

Faculté d'architecture, d'ingénierie architecturale, d'urbanisme (LOCI)

Univesrité catholique de Louvain (UCL).

Éditeur responsable : Le comité de rédaction, place du Levant, 1 - 1348 Louvain-la-Neuve

Comité de rédaction : Damien Claeys, Gauthier Coton, Pauline Fockedey,

Nicolas Lorent, Guillaume Vanneste

Conception graphique : Nicolas Lorent 


\title{
Gestion dynamique de la nature temporaire en Région bruxelloise
} Biodiv.Temp

\author{
Catherine Lembrée, Jonas Hanssens, \\ Aurélien Hucq, Fiorella Quadu - co-auteurs \\ Yves Hanin et Charles-Hubert Born - promoteurs de la recherche
}

\begin{abstract}
La recherche Biodiv.Temp est menée conjointement par le Centre de recherches et d'études pour l'action territoriale (CREAT) et le Séminaire de recherche en droit de l'environnement et de l'urbanisme (SERES) dans le cadre d'un financement Innoviris Anticipate. Cette recherche a pour objectif de fournir les outils juridiques et d'urbanisme nécessaires à l'établissement d'un maillage vert et bleu dynamique et cohérent tout en densifiant l'habitat en tenant compte des dynamiques temporelles et spatiales propres à la Région de Bruxelles-Capitale.
\end{abstract}

\section{Le contexte bruxellois}

Comme l'indiquent les documents de planification stratégique ${ }^{1}$ de la Région de Bruxelles-Capitale (RBC), la création de logements est un objectif majeur pour les prochaines années, sans pour autant négliger la préservation d'un cadre de ville le plus vert et durable. En effet, faisant face à une croissance démographique importante, le gouvernement bruxellois a la volonté d'augmenter l'offre en logements tout en assurant à chaque Bruxellois l'accès à un espace vert à moins de $400 \mathrm{~m}$ de son domicile ; $200 \mathrm{~m}$ si l'espace vert est inférieur à 1 ha $^{2}$. Si la Région bruxelloise est relativement verte, les espaces verts couvrant approximativement 8500 hectares $(50 \%$ de la superficie régionale), il existe des disparités importantes entre le centre de la région, peu végétalisé, et la périphérie à l'ouest et au nord où se concentrent les grandes zones de milieux ouverts ${ }^{3}$. De plus, seulement un tiers des espaces verts sont pleinement accessibles au public, ce qui correspond environ à $26 \mathrm{~m}^{2}$ par habitant $\mathrm{t}^{4}$.

Malgré la forte présence de nature et l'action du gouvernement pour lutter contre la diminution de la biodiversité, un nombre croissant d'espèces typiques des milieux ouverts continue de disparaitre. Les principales causes de ces disparitions : la pression de l'urbanisation et des activités humaines, ainsi que l'augmentation de la présence d'espèces exotiques.

L'enjeu est donc d'organiser la présence de la végétation en ville. II existe pour ce faire trois modalités assez usuelles. La première vise à développer des projets d'urbanisme et d'architecture intégrant la végétation et les cycles naturels à l'activité et à la présence humaine. L'autre modalité vise à identifier des réservoirs de biodiversité protégés de l'urbanisation. Entre ces différents espaces sont également présents des espaces corridors, de plus faibles surfaces, généralement linéaires, permettant de lier les zones protégées entre elles et avec les zones de nature partagée (parcs, jardins...).
Tant dans les projets intégrant la nature que dans les zones protégées, la gestion différenciée, de plus en plus courante, consiste à adapter les modes de gestion des espaces de nature aux caractéristiques et fonctions de chaque espace. Cette approche est le point de départ d'un changement de paradigme, permettant d'envisager la planification non pas statique, mais dynamique.

\section{Une nouvelle approche dynamique de la nature en ville}

Dans la continuité de ces réflexions, la recherche Biodiv.Temp vise à déployer une autre approche originale. En effet, les modalités citées précédemment impliquent des espaces stables et définis dans le temps. Or, il existe en RBC des espaces non bâtis temporaires, souvent à l'abandon, et sur lesquels s'installe une nature spontanée. Ces espaces délaissés, sans fonction définie, font partie intégrante de l'écosystème bruxellois, mais sont peu étudiés.

Très longtemps considérés comme des chancres urbains, des "verrues" à supprimer, ces espaces sont à nouveau considérés comme terrains à enjeux. Leur intérêt provient en particulier de leur caractère précaire autant pour les usages temporaires qui s'y installent parfois spontanément que pour la biodiversité qui s'y développe. Dans une région à l'espace limité, la temporalité de ces espaces pourrait être mise à profit dans une autre vision territoriale plus dynamique afin de répondre de façon complémentaire à la préservation de la biodiversité ou de l'accès aux espaces verts. 


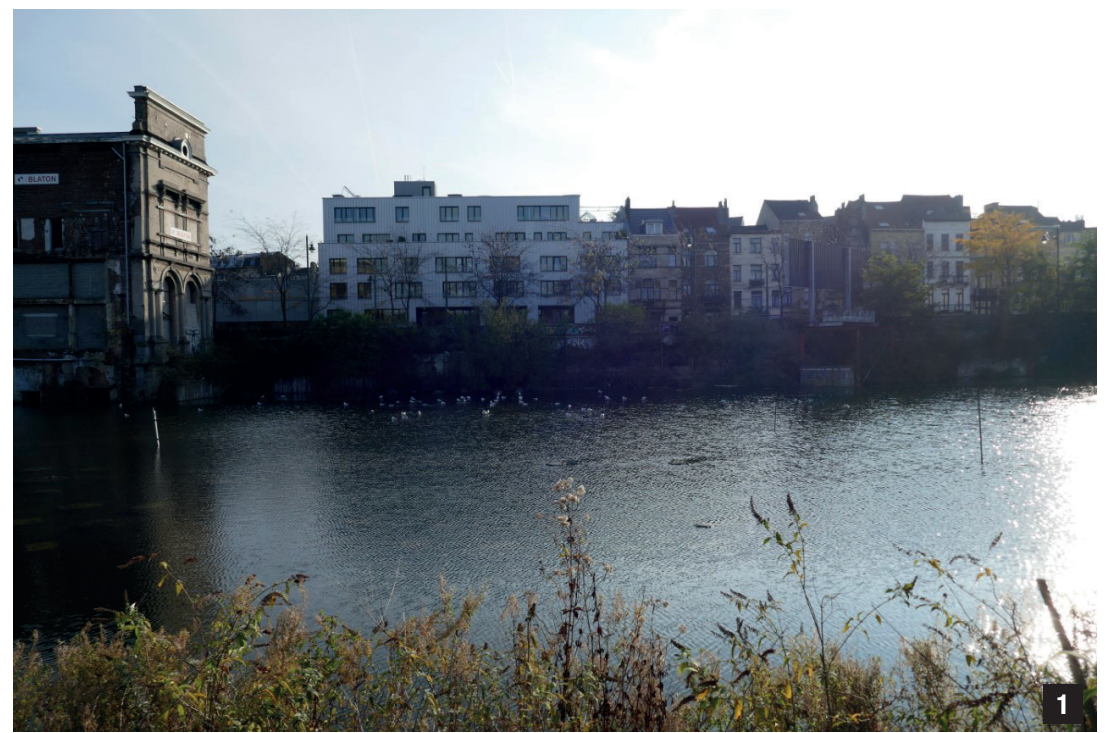

\section{Au cœur des mutations de la ville : les espaces délaissés}

Dans le cadre de la recherche, les espaces délaissés ont été définis comme des espaces artificialisés ou non qui sont majoritairement non bâtis et en friche, c'est-à-dire avec la présence de végétation dite spontanée : une végétation qui s'implante et croît sans intervention humaine sur un site. Ces espaces doivent également être en état de tiers foncier. Ce concept, défini par Marion Serre $^{5}$, reprend les espaces non bâtis qui présentent une inadéquation entre leur morphologie, leur statut et leurs usages $^{6}$. Nous pouvons citer comme exemple un terrain dont la propriété (statut) a été rendue floue par l'abandon, un terrain qui est le produit d'une mauvaise planification ou un terrain qui est squatté.

Enfin, notre étude se limite aux espaces urbanisables tels que fixés par le PRAS c'est-à-dire non affectés en zone verte. En ce sens, seuls les délaissés qui sont susceptibles d'être urbanisés à court ou moyen terme sont étudiés.

Les espaces délaissés étudiés ont pour caractéristique commune d'être temporaires. En effet, dans un territoire attractif comme Bruxelles, les espaces délaissés sont souvent réappropriés, formellement ou informellement pour finir par être remis en projet.

Dans ce cadre-là, différentes trajectoires peuvent être envisagées selon les trois temps de la friche décrits par Lauren Andres $^{8}$ et repris dans la figure 2 ci-dessous.
La figure montre les différentes trajectoires qui peuvent être prises durant le temps de veille. En effet, un temps d'abandon sans la présence humaine peut s'observer avant une réappropriation, comme parc, potager, squat... Dans certains cas, l'appropriation se fait directement, sans passer par une phase d'abandon. Enfin, les phases d'abandon et de réappropriation peuvent se succéder. Durant ce temps de veille, une nature temporaire peut s'installer. La nature temporaire se déplaçant, c'est l'existence éphémère de ces espaces qui permet un renouvellement et le maintien de ce type de milieu. L'originalité de la recherche est de considérer ce temps de veille comme partie intégrante du réseau écologique.

\section{Les services écosystémiques produits par la nature temporaire}

Le délaissé constitue donc généralement un écosystème jeune colonisé par des espèces herbacées pionnières, qui peut évoluer vers un stade arbustif, arborescent et enfin de forêt. La végétation à ce premier stade pionnier et éphémère est souvent qualifiée de nature temporaire, définie comme "le développement de nature dans une zone qui n'est pas initialement destinée à sa conservation et dont la destruction est programmée" 9 . La nature, qu'elle soit temporaire ou permanente, fournit des services écosystémiques. Ceux-ci sont définis comme "les avantages que la société humaine tire des écosystèmes"10. La figure 3 cidessous reprend les différents types de services écosystémiques existants.

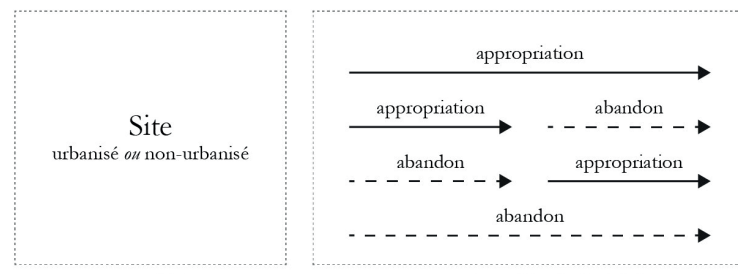

avant friche

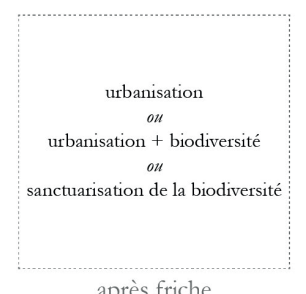

après friche
1 Exemple d'espace délaissé : le marais du Wiels (photo : Jonas Hanssens)

2 rajectoires des espaces délaissés source : Biodiv.Temp

5-Serre, M. (2017). Le Tiers Foncier Ressources, controverses et expérimentations - l'exemple de la ville de Marseille. Thèse de doctorat. Aix Marseille Université, Ecole Nationale Supérieure d'Architecture de Marseille, Marseille, France.

6 - La morphologie reprend les caractéristiques physiques des espaces, telles que la surface, la présence de végétation, l'artificialisation, etc. La morphologie donne la valeur de contexte du foncier. Le statut considère la valeur juridique du foncier, principalement la propriété. Enfin, l'usage décrit la fonction et l'usage, formel ou informel, de l'espace

(Serre, 2017).

8 - Andres, L. (2006). Temps de veille de la friche urbaine et diversité des processus d'appropriation : la Belle de Mai (Marseille) et le Flon (Lausanne). Géocarrefour, 81(2), 159-166.

6 - La morphologie reprend les caractéristiques physiques des espaces, telles que la surface, la présence de végétation, l'artificialisation, etc. La morphologie donne la valeur de contexte du foncier. Le statut considère la valeur juridique du foncier, principalement la propriété. Enfin, l'usage décrit la fonction et l'usage, formel ou informel, de l'espace (Serre, 2017).

9-Schoukens, H. (2016). Temporary nature: Is European nature conservation law ready for it? Life in Quarries.

10 - Millennium Ecosystem Assessment, 2005. Ecosystems and Human Well-being: Synthesis. Island Press, Washington, DC. 

source: Millennium Ecosystem Assessment, 2005 témiques - Source: Braat, L. Ten Brink, P. et al. (2008). The Cost of Policy Inaction: The case of not meeting the 2010 biodiversity target. Bruxelles: Commission Européenne, 22 p.

11 - Bonthoux, S., Brun, M., Di Pietro, F., Greulich, S., \& Bouché-Pillon, S. (2014). How can wastelands promote biodiversity in cities? A review. Landscape and Urban Planning, $132,79-88$

\section{ecosystem services}

\begin{tabular}{|c|c|}
\hline \multirow{3}{*}{$\begin{array}{l}\text { Supporting } \\
\text { nutrient cycling } \\
\text { soil formation } \\
\text { primary production } \\
\text {... }\end{array}$} & $\begin{array}{c}\text { Provisioning } \\
\text { food } \\
\text { fresh water } \\
\text { wood and fiber } \\
\text { fuel }\end{array}$ \\
\hline & $\begin{array}{l}\text { Regulating } \\
\text { climate regulation } \\
\text { food regulation } \\
\text { disease regulation } \\
\text { water purification }\end{array}$ \\
\hline & $\begin{array}{l}\text { Cultural } \\
\text { aesthetic } \\
\text { spiritual } \\
\text { educational } \\
\text { recreational }\end{array}$ \\
\hline
\end{tabular}

3

Le modèle des services écosystémiques est intéressant pour voir l'apport d'un espace à l'écosystème.

Dans l'étude de Braat, Ten Brink et al. (2008) la figure 4 montre que le lien entre la biodiversité, représentée dans l'étude par l'abondance moyenne des espèces (MSA), l'intensité d'utilisation du sol et la production de services écosystémiques. $\mathrm{Ce}$ graphique montre également qu'il n'existe pas de services écosystémiques propres aux espaces délaissés. Durant

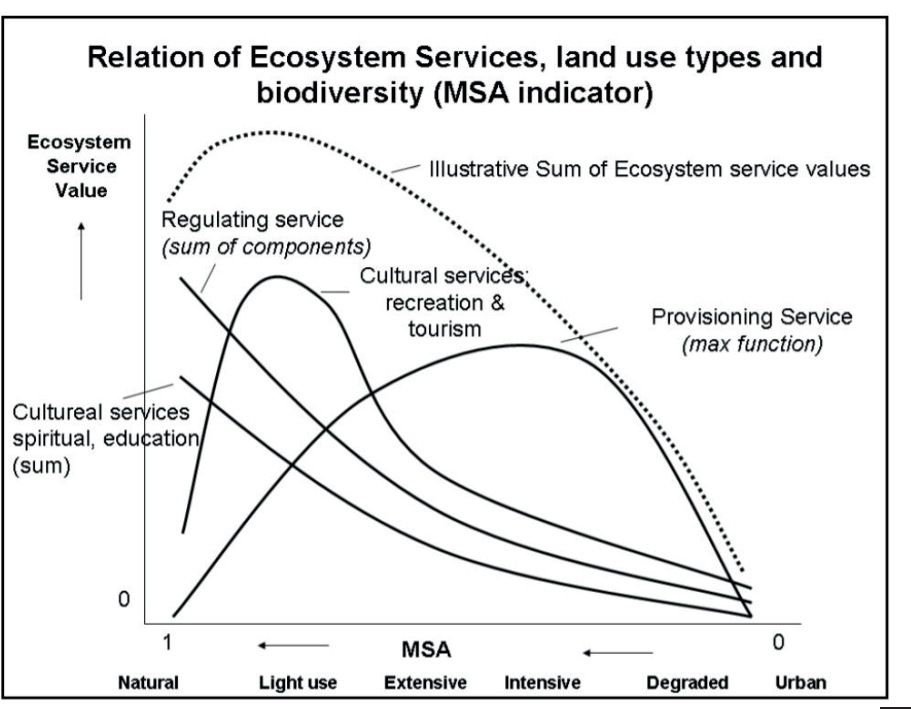

son temps de veille, correspondant à l'état de tiers foncier, l'espace peut être totalement délaissé ou avec une occupation humaine légère ou une succession de ces différents temps. À chacune de ces phases correspond une production spécifique de services écosystémiques. En effet, lors du temps d'abandon les services de régulation ou de support sont les plus élevés et diminuent au fur et à mesure de l'occupation humaine contrairement aux services culturels et aux services de production $(P)$ qui sont élevés lorsque l'Homme s'est approprié le territoire.

L'évaluation des services écosystémiques rendus par les délaissés permet de donner de la visibilité à la biodiversité et à la nature temporaire d'une part, mais souligne aussi l'importance de ces espaces dans les dynamiques associées au réseau écologique urbain. Nous avons identifié les facteurs qui conditionnent la production des services écosystémiques dans les délaissés ainsi que les indicateurs permettant de les mesurer.

L'approche utilisée consiste à analyser les caractéristiques propres aux délaissés qui influencent la biodiversité, c'està-dire la superficie, le sol, les occupations adjacentes, l'âge, le microclimat et la structure végétale ${ }^{11}$; les services écosystémiques qu'elles rendent; et les indicateurs permettant de les mesurer dans les écosystèmes urbains et identifiés par le Millenium Ecosystem Assessment.

La figure 5 ci-dessous comprend des exemples de services écosystémiques mesurables dans les délaissés et leurs variations selon la phase du temps de veille dans laquelle se trouve le délaissé.

\section{Des espaces en conflits juridiques}

Nous avons démontré la capacité des espaces délaissés à être multifonctionnels. Utilisés comme réserves foncières, comme refuges de biodiversité, comme espaces de nature informelle, ces délaissés sont dès lors des zones de conflits. Après avoir défini et cartographié ces espaces, puis avoir mesuré les services écosystémiques qu'ils rendent, le dernier objectif de la recherche Biodiv.Temp est d'étudier les possibilités juridiques de mobiliser des espaces délaissés pour y développer la biodiversité urbaine sans empêcher une urbanisation par la suite notamment en raison de la colonisation du site par des espèces protégées.

La mobilisation temporaire de terrains en friche à des fins de conservation de la nature dans l'attente de leur urbanisation peut être juridiquement appréhendée en deux temps. Le temps de veille correspond au laps de temps courant entre le moment du délaissement de l'espace et l'exécution des travaux d'urbanisation. L'enjeu de cette première phase est d'assurer juridiquement un développement temporaire de la nature sur l'espace considéré.

L'objectif est rempli tant que l'attitude du propriétaire permet à la nature de se développer. II importe peu, à cet égard, qu'il y prenne part activement, par des mesures de développement ou de res- 
services et disservices écosystémiques

avant occupation temporaire

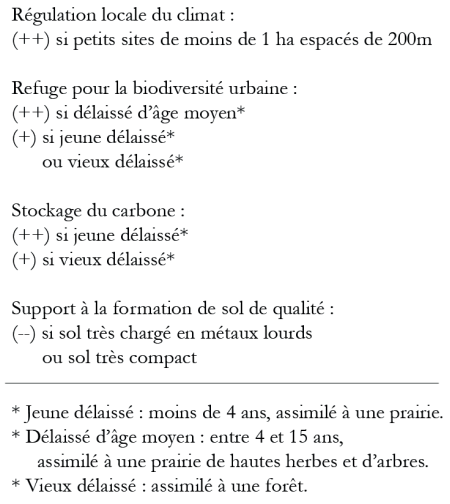

tauration de la nature, ou passivement, sans aucune intervention de sa part, en laissant les espèces coloniser spontanément son terrain. Notons que le statut des espèces pionnières rencontrées sur le terrain emporte des conséquences juridiques. Ainsi par exemple, si l'espace est colonisé par des espèces invasives au sens de l'Ordonnance "Nature"12, l'on pourrait y voir des troubles anormaux du voisinage emportant pour le propriétaire de l'espace en friche l'obligation de payer une juste et adéquate compensation aux propriétaires riverains dont le bien est envahi.

L'objectif de développement de la nature n'est en revanche pas rempli lorsque des mesures sont prises activement par le propriétaire pour éviter la "nature"13, particulièrement les espèces protégées, de façon à éviter tout problème lors de l'urbanisation du site. Les pouvoirs publics qui souhaitent passer outre cette réticence du propriétaire à développer temporairement la biodiversité sur un délaissé disposent alors d'un panel de différents outils juridiques que l'on peut distinguer selon l'atteinte faite aux droits du propriétaire. L'autorité publique peut acquérir la maîtrise du terrain concerné par une politique d'acquisition reposant sur des outils de politique foncière plus ou moins agressifs comme l'expropriation pour cause d'utilité publique ou un droit de préemption. L'autorité peut également contraindre le propriétaire à développer de la nature via une servitude légale d'utilité publique qui grève son fond. Enfin, l'autorité publique peut encadrer voire promouvoir une telle initiative par la conclusion de conventions ou l'octroi de subventions.

Après un temps de veille, la deuxième phase correspond au commencement d'exécution des travaux d'urbanisation. L'enjeu de cette phase est d'assurer juridiquement la légalité de la destruction de la "nature temporaire " ${ }^{14}$ présente sur le site. De deux choses l'une.

\section{pendant occupation temporaire}

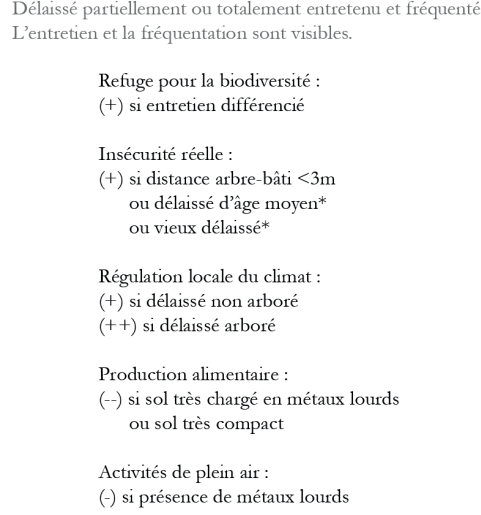

Si une espèce protégée est présente sur le site, une dérogation devra être en principe obtenue auprès de l'autorité compétente ${ }^{15}$. En l'absence d'une telle dérogation, la poursuite des travaux est illicite. À l'inverse, si aucune espèce protégée n'est présente sur le site, alors les travaux seront en principe admis juridiquement pour autant que la destruction de la nature n'affecte pas l'intégrité d'un site voisin protégé en vertu de l'Ordonnance "Nature", laquelle, le cas échéant, emporte la nécessité d'une dérogation ${ }^{16}$.

\section{Conclusion}

En conclusion, la recherche Biodiv. Temp a pour objectif de caractériser les espaces délaissés en RBC, de comprendre les dynamiques de mutation de ces espaces ainsi que les dynamiques végétales qui y prennent court. Cette caractérisation permet de démontrer l'importance de prendre en compte la temporalité des espaces dans le réseau écologique bruxellois pour la fourniture de certains services écosystémiques. Ensuite, la recherche s'attache à la mise en place d'un cadre juridique et de planification permettant de gérer cette temporalité et les conflits qui en découlent.
5 Services et disservices écosystémiques principalement produits par un délaissé urbain - source Biodiv.Temp

12 - Définie comme "espèce exotique qui a tendance à se propager ou à se répandre en grand nombre, de manière excessive ou menaçante pour la préservation de la diversité biologique". Art. $3,45^{\circ}$, Ordonnance du ler $^{\text {er }}$ ars 2012 relative à la conservation de la nature, M.B., 16 mars 2012, p. 16.017.

13 - L’exemple généralement pris est celui des pesticides afin d'éviter toute végétation.

14-Du moins celle qui n'est pas prévue dans le projet d'urbanisation. Rappelons également que le PRAS impose, sans aucune dérogation possible, que certains projets d'urbanisme prévoient le maintien ou la réalisation d'une superficie minimum d'espaces verts (PRAS, 0.2, al. 2).

15-Art. 83 et s., Ordonnance "Nature".

16-Art. 64-65, Ordonnance "Nature". 